\title{
Effects of short-term plasticity in early olfactory information processing in Drosophila
}

\author{
Yuxuan Liu ${ }^{1}$, Qianyi $\mathrm{Li}^{2,5}$, Chao Tang ${ }^{1,3,4}$, Shanshan Qin ${ }^{3,6, \bigotimes}$, and Yuhai $\mathrm{Tu}^{7, \mathbb{}}$ \\ ${ }^{1}$ School of Physics, Peking University, Beijing 100871, China; \\ ${ }^{2}$ Integrated Science Program, Yuanpei College, Peking University, Beijing 100871, China; \\ ${ }^{3}$ Center for Quantitative Biology, Peking University, Beijing 100871, China; \\ ${ }^{4}$ Peking-Tsinghua Center for Life Sciences, Peking University, Beijing 10087, China; \\ ${ }^{5}$ Biophysics Graduate Program, Harvard University, Cambridge, MA 02138; \\ ${ }^{6}$ John A. Paulson School of Engineering and Applied Sciences, Harvard University, Cambridge, MA 02138; \\ ${ }^{7}$ IBM T. J. Watson Research Center, Yorktown Heights, NY 10598
}

\begin{abstract}
In Drosophila, olfactory information received by the olfactory receptor neurons (ORNs) is first processed by an incoherent feed forward neural circuit in the antennal lobe (AL) that consists of ORNs (input), the inhibitory local neurons (LNs), and projection neurons (PNs). This "early" olfactory information process has two important characteristics. First, response of a PN to its cognate ORN is normalized by the overall activity of other ORNs, a phenomenon termed "divisive normalization". Second, PNs respond strongly to the onset of ORN activities, but they adapt to prolonged or continuously increasing inputs. Despite the importance of these characteristics for learning and memory, their underlying mechanism remains not fully understood. Here, we develop a circuit model for describing the ORN-LN-PN dynamics by including key features of neuron-neuron interactions, in particular shortterm plasticity (STP) and presynaptic inhibition (PI). Our model shows that STP is critical in shaping PN's steady-state response properties. By fitting our model to experimental data quantitatively, we found that strong and balanced short-term facilitation (STF) and short-term depression (STD) in STP is crucial for the observed nonlinear divisive normalization in Drosophila. By comparing our model with the observed adaptive response to time-varying signals quantitatively, we find that both STP and PI contribute to the highly adaptive response with the latter being the dominant factor for a better fit with experimental data. Our model not only helps reveal the mechanisms underlying two main characteristics of the early olfactory process, it can also be used to predict the PN responses to arbitrary time-dependent signals and to infer microscopic properties of the circuit (such as the strengths of STF and STD) from the measured input-output relation.
\end{abstract}

Short-term plasticity | Presynaptic inhibition |Circuit model | Olfactory system | Drosophila

\section{Introduction}

Sensory systems have evolved different strategies to efficiently represent and process physiologically relevant stimuli in the presence of various biophysical constraints. For example, the olfactory system is confronted with the challenge that there are numerous odors each consisting of multiple volatile molecules with a wide range of concentrations. Yet the olfactory system possesses a remarkable ability to detect and discriminate odors using a relatively small repertoire of odor receptors (ORs) through a combinatorial code, i.e., each odorant is sensed by multiple receptors and each receptor can be activated by many odorants (1-3).

The functional organization of the olfactory systems across different species is highly conserved (4-6). In both insects and vertebrates, an olfactory receptor neuron (ORN) typically expresses only one type of OR. ORNs that express the same OR converge to the same glomerulus in the olfactory bulb (vertebrates) or antennal lobe (AL, insects). In Drosophila, peripheral odor information is processed in AL before transmitted to higher brain areas by projection neurons (PNs) (7, 8). Each PN typically innervates one glomerulus. The transfer function between ORN and PN is a saturating nonlinear function (9-11), i.e., a small ORN input is disproportionally amplified while a strong input saturates the response. Lateral inhibition by the local interneurons (LNs) in the AL increases the level of ORN input needed to drive PNs to saturation, the strength of inhibition scales with the total forward input to the AL, a phenomenon called "divisive normalization" $(12,13)$, which is beneficial for efficient odor coding $(10,14)$.

Airborne odors are intermittent and have complex spatio-temporal profiles $(15,16)$. The ability to detect and respond to temporal variation of odors is crucial for successful odor-guided navigation (17-19). This is partially achieved by the response properties of PNs to time-dependent inputs from ORNs. For example, PNs respond transiently to a step-function like ORN input and fall back to low firing rates for the prolonged input, showing highly adaptive response. For more complex time-dependent ORN input, the response of PNs depends on both the ORN firing rate and its rate of change (20-22). These properties are important for detecting and tracking natural odor stimuli. Although a phenomenological linear-nonlinear model was proposed to fit experimental data (20), a mechanistic understanding of how the ORN-PN-LN circuit in AL leads to the adaptive response is still missing. 
The aim of this study is to understand both "divisive normalization" and the adaptive response to time-varying stimuli of PNs by modeling the dynamics of the AL neural circuit. Previous studies showed that synapses between ORNs and PNs exhibit strong short-term plasticity (STP) (9, 23), which is a form of fast activity-dependent modulation of synaptic strength (24-28). Furthermore, inhibition by LNs is due to presynaptic inhibition (PI) at the axon terminal of ORNs (10). In the rest of the paper, we first develop a circuit model of the Drosophila AL that incorporates both STP and PI. Then, with our analytical results and numerical simulations, we show that STP is essential for the observed highly nonlinear divisive normalization; and both STP and PI determine the adaptive response observed in experiments.

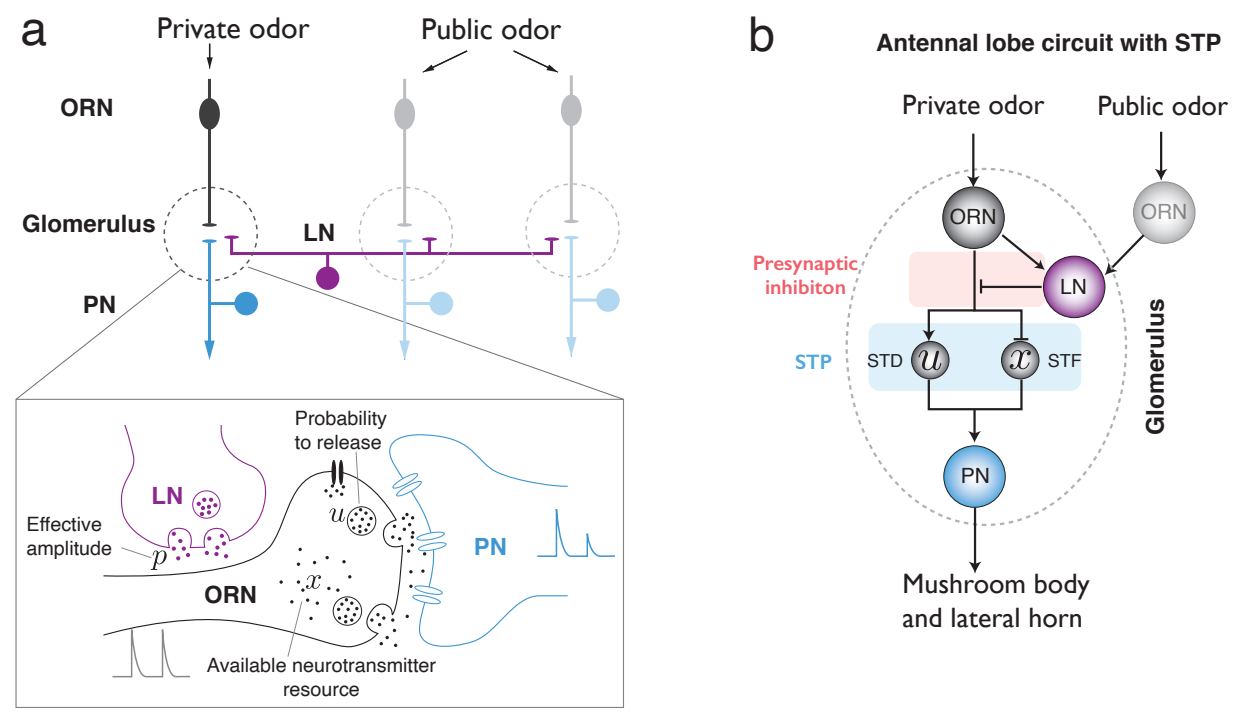

Fig. 1. Illustration of the antennal lobe (AL) circuit model. (a) Anatomy of the peripheral olfactory system in Drosophila. Olfactory receptor neurons (ORNs) that express the same type olfactory receptors innervate the same glomeurlus (dashed region) in AL. Most projection neurons (PNs) send dendrites into one glomerulus and receive synaptic input from the ORN. Although each glomerulus might be innervated by different PNs, only one PN is shown. Glomeuruli are laterally connected by local neurons (LNs,magenta), which interact with ORNs and PNs. A private odor only activates a specific type of ORNs, while a public odor activates a large number of ORNs that innervate different glomeruli. Lower: close-up of the synaptic interaction between ORN, PN and LN. Both PI and STP are considered in our model. (b) Schematics of the AL circuit with STP effect and PI mediated by LNs.

\section{A circuit model of the peripheral olfactory system with STP}

There are around 50 types of ORNs in Drosophila melanogaster, each of them expresses one OR. ORNs that express the same OR converge to the same glomerulus in the AL. A given odor typically activates several types of ORNs, hence different glomeruli. Each PN innervates one glomeulus and projects to higher brain areas like mushroom body and lateral horn. Since most LNs in AL are GABAergic, we will only consider inhibitory interneurons. Although LNs have distinct morphologies, innervation patterns, and response dynamics to odors (29-31), for the purpose of this study, we do not differentiate them in our model. Generally speaking, LNs innervate different glomeruli and target the butons of ORN axons, forming presynatpic inhibition (Fig.1a). We consider the simplified neural circuit of ORN-PN-LN in the antennal lobe, which forms an incoherent feedforward loop (IFFL) as shown in Fig.1b.

For simplicity, we assume that the firing rate $\left(R_{\mathrm{PN}(\mathrm{LN})}\right)$ of the $\mathrm{PN}(\mathrm{LN})$ is proportional to its conductance $\left(g_{\mathrm{PN}(\mathrm{LN})}\right)(32)$ : $R_{\mathrm{PN}(\mathrm{LN})}=k g_{\mathrm{PN}(\mathrm{LN})}$ with a constant $k$, which is verified in the leaky integrate-and-fire model (see numerical validation in Fig.S1 in Supplementary Material (SM)). Dynamics of PN (LN) conductance $g_{\mathrm{PN}(\mathrm{LN})}$ can be written as:

$$
\begin{gathered}
\frac{d g_{\mathrm{PN}}}{d t}=-\frac{g_{\mathrm{PN}}}{\tau_{E}}+\omega^{E E} u^{+} x p R, \\
\frac{d g_{\mathrm{LN}}}{d t}=-\frac{g_{\mathrm{LN}}}{\tau_{E}}+\omega^{I E} \sum_{j} R_{j},
\end{gathered}
$$

where $R$ is the firing rate of the cognate ORN that responds to a particular (private) odorant. The sum $\sum_{j}$ in Eq. (2) is over all ORNs connected to the LN, including non-cognate ORNs that respond only to public odorant(s). The timescale $\tau_{E}$ is the relaxation time of conductance. $\omega^{E E}$ and $\omega^{I E}$ are synaptic weights of the synapses from ORN to PN and LN respectively, which we assume to be homogeneous among different ORNs.

The effect of PI is modeled by Eq. (1) with a (dimensionless) variable $0<p<1$ that represents reduction of the effective ORN firing rate due to presynaptic inhibition by LN. The dynamics of $p$, simplified from previous studies $(33,34)$, is modeled as:

$$
\tau_{p} \frac{d p}{d t}=-p+\frac{1}{1+\rho R_{L N}}
$$


where $\rho$ is a constant and $\tau_{p}$ is the relaxation time of $p$. In the limit $\tau_{p} \ll \tau_{E}$, we can use the quasi-steady state approximation $p \approx \frac{1}{1+\rho R_{\mathrm{LN}}}$, which suggests that $p$ decreases with $R_{\mathrm{LN}}$.

The effect of STP can be separated into short term facilitation (STF) and short term depression (STD), which are modeled by $u^{+}$and $x$ in Eq. (1) respectively. Following from previous work (35), we denote $u^{-}\left(u^{+}\right)$as the releasing probability just before (after) the arrival of a presynaptic spike; and $x$ as the fraction of available neurotransmitters (Fig. 1a). Applying the mean-field model for STP (35), we have the following dynamics for $u^{ \pm}$and $x$ :

$$
\begin{aligned}
\frac{d x}{d t} & =\frac{1-x}{\tau_{D}}-x u^{+} p R, \\
\frac{d u^{-}}{d t} & =-\frac{u^{-}}{\tau_{F}}+U\left(1-u^{-}\right) p R,
\end{aligned}
$$

where $u^{+}=u^{-}+U\left(1-u^{-}\right)$with $U$ as the increment in release probability after each spike. Without any presynaptic firing ( $R=0$ ), we have $x=1, u^{-}=0$, and $u^{+}=U$ at steady state. With presynaptic firing, $x$ decreases and $u^{+}$increases before their steady state values are recovered with time constants $\tau_{D}$ and $\tau_{F}$ respectively. Therefore, the strength of STD and STF can be measured by their normalized recovery time (dimensionless), $S_{F}=\frac{\tau_{F}}{\tau_{E}}$ and $S_{D}=\frac{\tau_{D}}{\tau_{E}}$. The longer the recovery time, the stronger the effect.

\section{Results}

We use the neural circuit model proposed in the previous section to describe and explain several response properties of PNs including divisive normalization for constant (steady state) inputs and adaptive response to time-varying inputs. In both cases, we compare our model results with existing experiments and focus on understanding the effects of STP and PI on the observed behaviors.

STP is crucial for the observed nonlinear divisive normalization. For any input (cognate ORN with firing rate $R$ ) to the peripheral olfactory system, its output, i.e., the firing rate of the cognate PN can be computed by solving Eqs. (1)-(5). For a constant input (fixed firing rates of all ORNs), the steady state PN response can be determined analytically:

$$
R_{P N}^{*}(R)=\frac{k \tau_{E} \omega^{E E} U R\left(\theta+\tau_{F} R\right)}{\theta^{2}+\theta\left(\tau_{F}+\tau_{D}\right) U R+\tau_{D} \tau_{F} U R^{2}},
$$

where $\theta \equiv 1+A \sum_{j} R_{j}$ with $A \equiv k \rho \omega^{I E} \tau_{E}$, and $\sum_{j} R_{j}=R+\hat{R}_{\text {pub }}$ with $\hat{R}_{\text {pub }}$ denoting the total input from public ORNs.

In the absence of STP, i.e., when $\tau_{D} \rightarrow 0$ and $\tau_{F} \rightarrow 0, x=1$ and $u^{+}=U$ remain constant, the PN response Eq. (6) reduces to:

$$
R_{P N}^{*}(R)=k \tau_{E} \omega^{E E} U \frac{R}{1+A \sum R_{j}},
$$

where both the numerator and denominator depend linearly on $R$. Eq. (7) can thus be called "linear" divisive normalization. In the presence of STP, i.e., when $\tau_{F} \neq 0$ and $\tau_{D} \neq 0$, both the denominator and the numerator in Eq. (6) are nonlinear in $R$, which we call "nonlinear" divisive normalization.

The PN response curve exhibits a sigmoidal shape that can be characterized by three parameters: the maximum response $R_{\mathrm{PN}}^{\max } \equiv R_{\mathrm{PN}}^{*}(R=\infty)=\frac{k \tau_{E} \omega^{E E} U\left(A+\tau_{F}\right)}{A^{2}+A\left(\tau_{F}+\tau_{D}\right) U+U \tau_{F} \tau_{D}}$; the half maximum input $R_{1 / 2}$ defined as $R_{P N}^{*}\left(R_{1 / 2}\right)=R_{\mathrm{PN}}^{\max } / 2$; and an effective Hill coefficient $\left.\gamma \equiv 2 \frac{d \ln \left(R_{\mathrm{PN}}^{*}\right)}{d \ln (R)}\right|_{R_{1 / 2}}$. From Eq. (7), we have $\gamma=1$ in the absence of STP. In the presence of STP, we have $\gamma>1$, which can be used to characterize the gain of the response. In Fig. 2a\&b, the PN response function Eq. (6) with different STP strengths are shown. As expected, for a given input $R$, STF enhances the response while STD suppresses it with the latter having a stronger effect. The dependence of the gain $\gamma$ on the STP strengths $S_{D}$ and $S_{F}$ are shown in Fig. 2c. Interestingly, the gain parameter $\gamma$ is enhanced by both STD and STF. Larger values of $\gamma$ are reached by having roughly the same STD and STF strengths (dotted line in Fig. 2c).

As shown in Eq. (6), the PN response is suppressed by the firing of LN, which can be activated by many ORNs besides the cognate ORN. This introduces lateral inhibition and reduces the response to a private odorant in the presence of public odorants (Fig.2d). This divisive normalization effect was fitted by a Hill function in Olsen et al (12):

$$
R_{P N} \approx R_{\max } \frac{R^{\gamma}}{R^{\gamma}+K_{1 / 2}^{\gamma}+\sigma^{\gamma}},
$$

where $\sigma$ is assumed to be proportional to the sum of the firing rates of non-cognate ORNs; $R_{\max }$ is the maximum PN firing rate; $K_{1 / 2}$ is the firing rate of ORN at which PN has the half maximum response when $\sigma=0, \gamma$ is the Hill coefficient. In (12), the authors were able to fit their experimental data with $\gamma \approx 1.5$. 

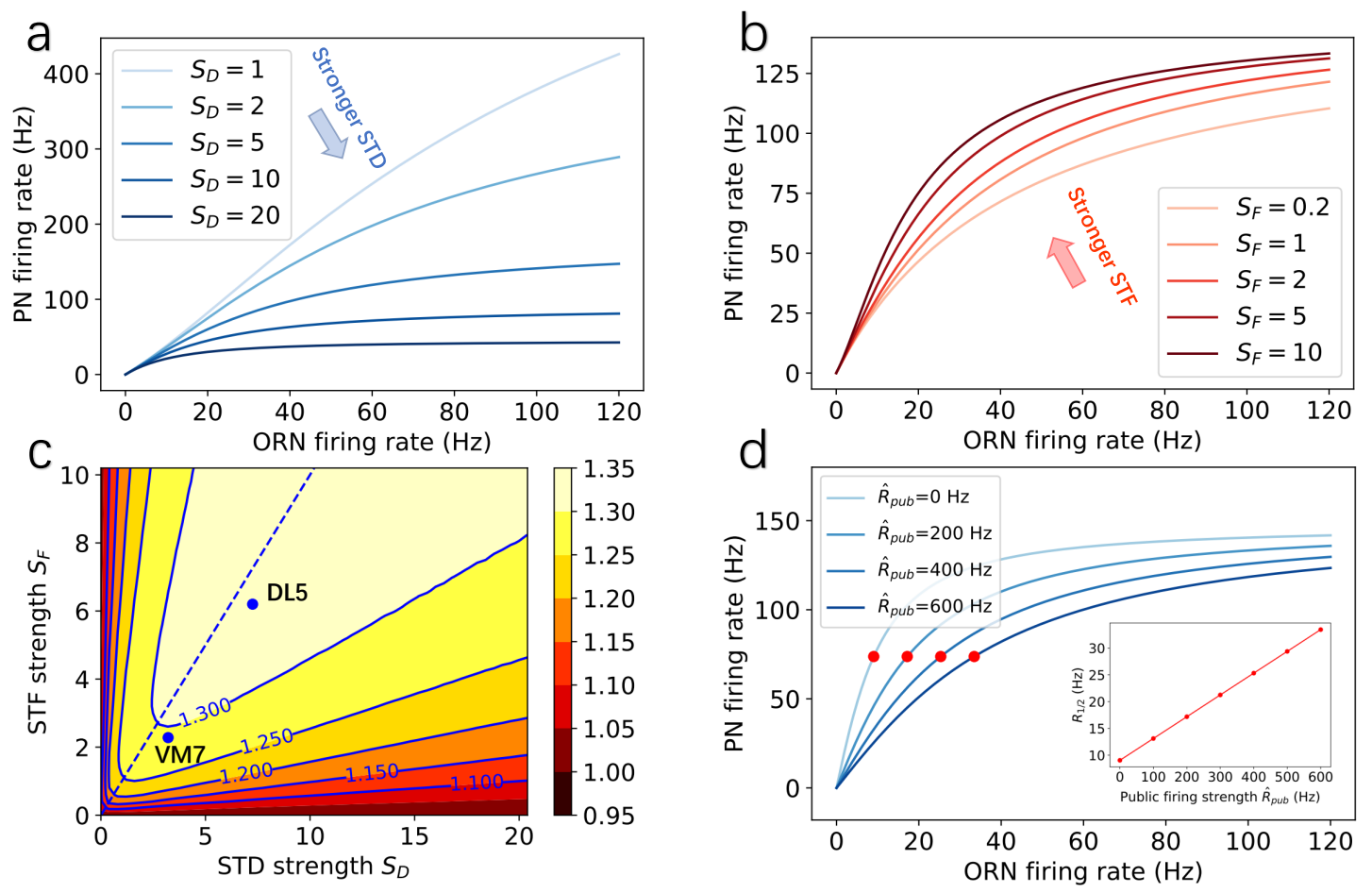

Fig. 2. The effects of STP (STF and STD) on the nonlinear divisive normalization effect in PNs' responses. (a,b) Steady state responses of PN to different cognate ORN firing rates for (a) different STD strengths $S_{D}$ and (b) different STF strengths $S_{F}$. In (a) $S_{F}=2$, in (b) $S_{D}=6$. (c) The dependence of the effective Hill coefficient $\gamma$ on the STF strength $\left(S_{F}\right)$ and the STD strength $\left(S_{D}\right)$. The values of $S_{D}$ and $S_{F}$ used in fitting the experimental data for DL5 and VM7 (see Fig. 3) are also shown in the figure. The dotted line corresponds to perfectly balanced STF and STD strength: $S_{D}=S_{F}$. (d) Steady state responses of PN to cognate ORN firing rates in the presence of different public firing rates $\hat{R}_{\text {pub }}$. Red dots mark ORN firing rates at the half maximum firing of PNs $\left(R_{1 / 2}\right)$. The inset shows that $R_{1 / 2}$ increases linearly with $\hat{R}_{\text {pub }}$. Other model parameters used here are: $U=0.24, \hat{R}_{\mathrm{pub}}=500 \mathrm{~Hz}, \mathrm{k}=5 \mathrm{~Hz} / \mathrm{nS}, \omega^{\mathrm{EE}}=180 \mathrm{nS}, \omega^{\mathrm{IE}}=10 \mathrm{nS}, \tau_{\mathrm{E}}=50 \mathrm{~ms}, \rho=0.0018$.

From our model, this experimental observation value of $\gamma(>1)$ suggests the existence of strong STP effects. To verify this hypothesis, we fit the experimental results reported in (12) using Eq. (6) from our model. In a typical experiment, PN responses to a private odorant (which activates only the cognate ORN) with a background of different concentrations of a public odorant (presumably activates many other ORNs) was measured. As shown in Fig. 3, each color represents a different concentration of the public odorant, the exact firing rate is not available from the experiment, but was assumed to be proportional to the measured local field potential (LFP). In our model this is fitted by adjusting the value of total firing rate of public ORNs $\hat{R}_{\text {pub. }}$.
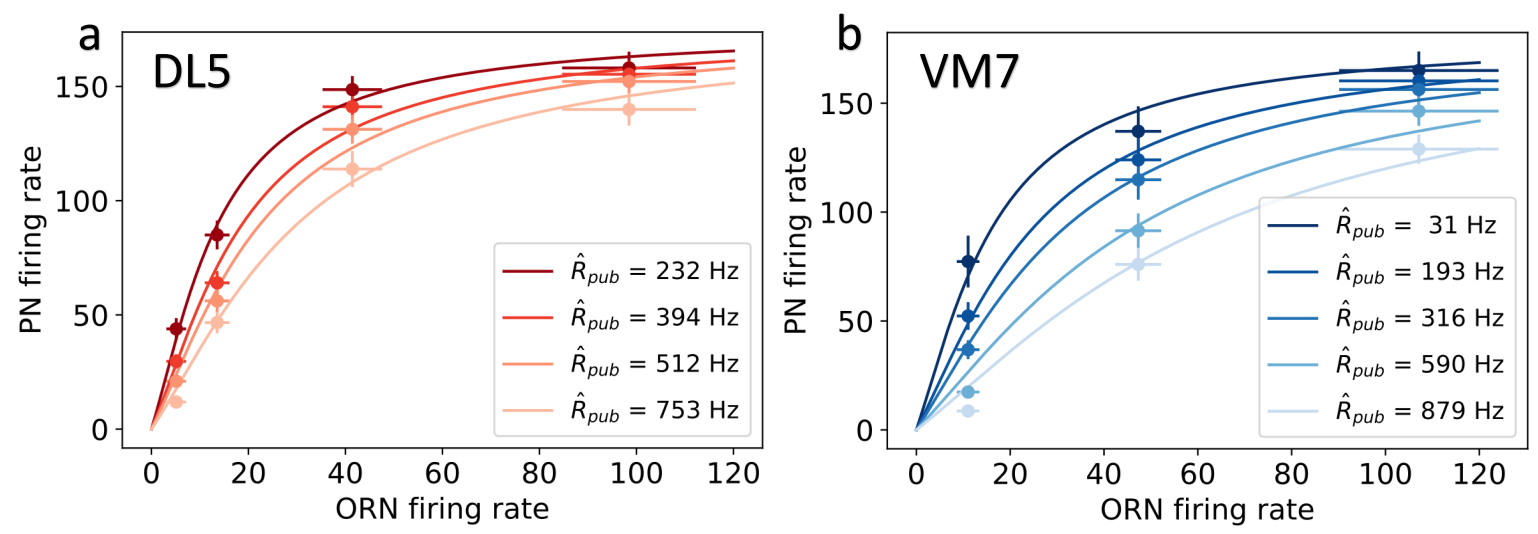

Fig. 3. The circuit model with STP and PI can fit experimental data exhibiting the nonlinear divisive normalization effect in a PN's response. Different shades of colors indicate different strengths of lateral inhibition due to different public ORN firing rates. PNs that inervate the (a) DL5 and (b) VM7 glomeruli are compared. Experimental data are from (12). The best-fit parameters shown in Table 1 are within physiological ranges.

Our model fits well with the measured responses of the PNs that innervate either DL5 or VM7 glomeruli for different levels of public odorant concentrations. The best-fit parameter sets for DL5 PN and VM7 PN are given in Table 1. While most of the parameters for DL5 and VM7 remain approximately the same, there are some differences that are informative. In particular, the two STP timescales $\left(\tau_{D}\right.$ and $\left.\tau_{F}\right)$ and the lateral inhibition strength $\rho$ are intrinsic properties of the glomerulus and are thus 
Table 1. Parameters in our model used for Fig. 3

\begin{tabular}{cccc}
\hline Parameter & Meaning & DL5 & VM7 \\
\hline $\mathrm{k}$ & linear coefficient from conductance to firing rate & $5 \mathrm{~Hz} / \mathrm{nS}$ & $5 \mathrm{~Hz} / \mathrm{nS}$ \\
$\tau_{E}$ & time constant for excitatory synapse & $50 \mathrm{~ms}$ & $50 \mathrm{~ms}$ \\
$\omega^{E E}$ & synaptic weight from ORN to PN & $247 \mathrm{nS}$ & $121 \mathrm{nS}$ \\
$\omega^{I E}$ & synaptic weight from ORN to LN & $10 \mathrm{nS}$ & $10 \mathrm{nS}$ \\
$\rho$ & intrinsic strength for presynaptic inhibition & 0.02 & 0.015 \\
$\mathrm{U}$ & increase in release probability for faciliation & 0.24 & 0.24 \\
$\tau_{D}$ & time constant for STD & $345 \mathrm{~ms}$ & $161 \mathrm{~ms}$ \\
$\tau_{F}$ & time constant for STF & $309 \mathrm{~ms}$ & $114 \mathrm{~ms}$ \\
\hline
\end{tabular}

expected to be different. Our model results indicate that both DL5 and VM7 have strong STP effects. Quantitatively, both STD and STF strength are stronger in DL5 than those in VM7, however, the relative strengths of STD and STF, $r \equiv \tau_{D} / \tau_{F}$, remain roughly the same for DL5 $(r \approx 1.1)$ and VM7 $(r \approx 1.4)$, which indicates that a strong and balanced STD and STF is responsible for the observed nonlinear divisive normalization in both VM7 and DL5. Both timescales $\left(\tau_{D}\right.$ and $\left.\tau_{F}\right)$ obtained from our model fitting are consistent with the range of these timescales measured in experiments (36). We also find that lateral inhibition is slightly stronger in DL5 than that in VM7.

Adaptive response to time-varying signals and its circuit origin. Odors in the environment are highly intermittent and dynamic $(15,16)$. The ability to detect and respond to temporal variation of odor stimuli is crucial for the survival of many animals. Kim et al (20) studied the responses of PNs to different time-dependent ORN signals that follow a triangle-shaped temporal pattern with different peak times. The responses of PNs were "asymmetric", with a faster rising phase, followed by a plateau and a slower decaying phase, depending on the rate of change in ORN's firing rate profile (see Fig. 3 in (20)). Here, we use our model to explain the response patterns to these time-dependent signals.

We start by considering the response of PNs to a signal that increases linearly with time, i.e., $R(t)=K t$. In the short time limit $t \ll \tau_{p}$, PI is negligible so the reduction factor $p \approx 1$ (see Eq. (3)). As a result, the response is linearly proportional to the input $R_{\mathrm{PN}}(t) \approx k \tau_{E} \omega^{E E} U K t$. For longer time $t \gg \tau_{p}, p \approx(A R(t))^{-1}$ decreases inversely proportional to the input $R(t)$ which reduces the effective input $p R(t)$. In fact, the effective input can be approximated as (see SM for detailed derivation):

$$
R_{\mathrm{eff}} \equiv p R(t) \approx \frac{K t}{A K\left(t-\tau_{E}\right)+1}-\frac{A K^{2} \tau_{E} t}{1-A K \tau_{E}} \cdot e^{-\frac{t}{\tau_{p}}}
$$

In the long time limit $t \gg \tau_{E}, \tau_{p}$, the system adapts by adjusting the inhibition factor $p$ so that the effective input reaches a constant $R_{\mathrm{eff}}^{*}=A^{-1}=\left(k \rho \omega^{I E} \tau_{E}\right)^{-1}$ as $t \rightarrow \infty$, which is independent of the input. The corresponding adapted response can thus be determined analytically:

$$
R_{\mathrm{PN}}^{*} \approx \frac{k \tau_{E} \omega^{E E} U R_{\mathrm{eff}}^{*}\left(1+\tau_{F} R_{\mathrm{eff}}^{*}\right)}{1+U R_{\mathrm{eff}}^{*}\left(\tau_{F}+\tau_{D}\right)+U \tau_{D} \tau_{F} R_{\mathrm{eff}}^{*}},
$$

which takes exactly the same form as the response to an effective time-independent signal $R_{\text {eff }}^{*}$ as described in Eq. (6). This is supported by numerical simulation as shown in Fig. 4. The effect of PI is crucial for canceling out the increasing signal, which results to a constant effective input $R_{\mathrm{eff}}^{*}$. From Eq. (10), it is clear that STP also affects the adapted response $R_{\mathrm{PN}}^{*}$ in the same way as it affects the response to a constant signal, i.e., STF enhances the adapted response and STD suppresses it.

We now study the PN responses to triangle-shaped input signals similar to those used in experiments (20) with our model. As shown in Fig. 5a, the general response dynamics follow closely the experimentally observed behaviors. During the rising phase of the input signal, the PN response activity reaches its peak in a timescale that depends on the rate of change $(K)$ of the input signal. For small $K$ shown as the purple line in Fig. 5a, the PN response reaches a plateau before the input reaches its peak due to the adaptive effect of presynaptic inhibition described above. The shaded region in Fig. 5a (lower panel) shows the range of the experimentally observed plateau consistent with the model result (purple line). Note that for higher $K$ the plateau activities depend on the rates of change in the input signals because they do not have enough time to reach the adapted value. In the descending phase of the input signal, the PN activity decreases following the input.

Quantitatively, the PN response can be described by two parameters: the peak response, which is defined as the PN activity at the peak time of the input signal, and the time to reach the peak response. In Fig. 5b\&c, we show that the results for these quantities measured from our model are in excellent agreement with those obtained from experiments (20).

In principle, STP (specifically STD) alone can also lead to adaptive responses. However, we find that the adaptive response dynamics to time-varying signals observed in experiments (20) is more strongly affected by PI. This can be seen by comparing PN's response to triangle-shaped input in four model variants: the standard model used in Fig. 5 with strong PI and moderate STP, a model with PI only without STP $\left(S_{D}=S_{F}=0\right)$, and a model with only STP without PI $(\rho=0)$. As shown in Fig. S2 


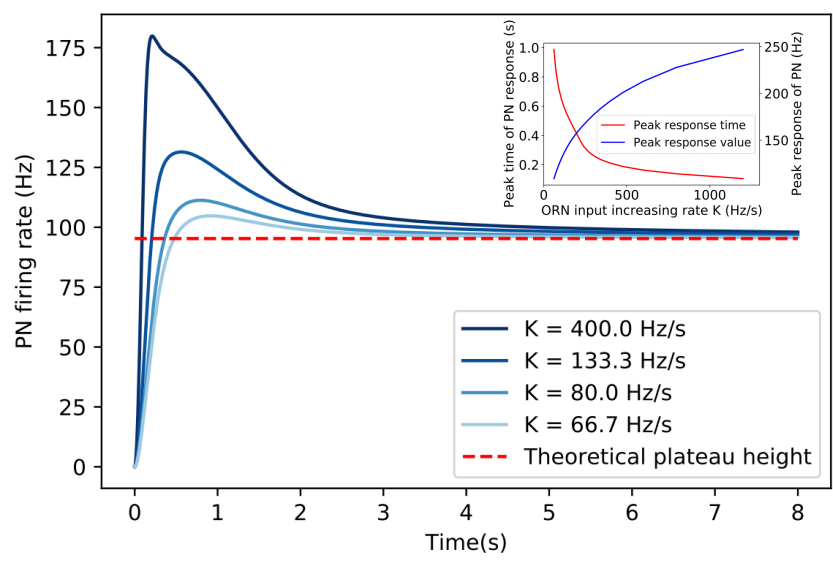

Fig. 4. The responses of $\mathrm{PN}$ to linearly increasing inputs (ORN firing rate) with different increasing rate $K$. The adapted responses are independent of the increasing rate $(K)$ of the input. Dashed line marks the asymptotic response when $t \rightarrow \infty$ as predicted by Eq. (10). The inset shows how the peak time and peak value of PN response depend on the increasing rate $(K)$.
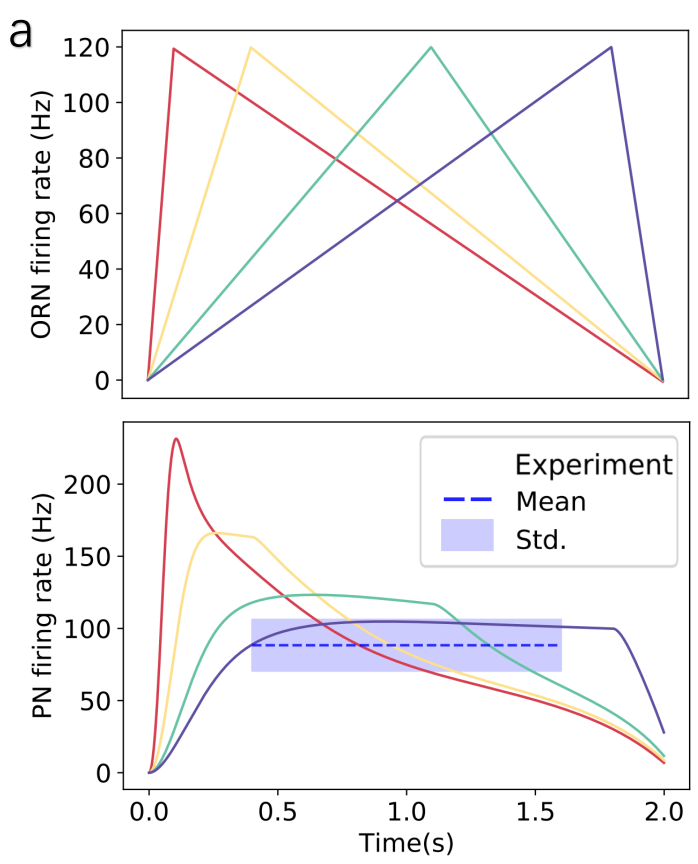
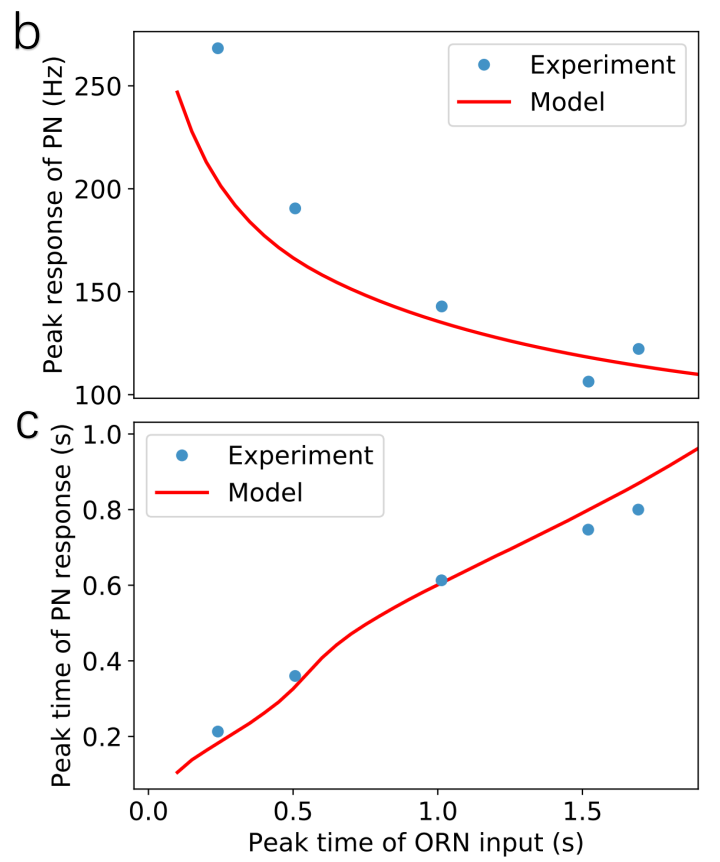

Fig. 5. Adaptive responses of $P N$ to triangle-shaped inputs (ORN spike rates). (a) Upper panel: Simulated triangle-shaped ORNs firing rates with different increasing rates in the rising phase. The peak inputs (ORN firing rates) are the same for all cases. Lower panel: responses of PNs to triangle-shaped ORNs inputs. For slow and medium increasing inputs, PNs reach plateau responses. The dotted line shows the average plateau response of PN estimated from experimental data with the shaded region indicating the standard derivation. (b) The peak responses of PNs increase with the rates of change in input signals. Our model result (solid line) agrees well with the experiment (dots). (c) PN's response reaches a peak earlier than that of the input signal. The model result (solid line) has an excellent agreement with the experiment (dots). Experimental results are from (20). Parameters used in the model: $U=0.24, R_{\text {pub }}=0 \mathrm{~Hz}, \mathrm{k}=5 \mathrm{~Hz} / \mathrm{nS}, \omega^{\mathrm{EE}}=75 \mathrm{nS}, \omega^{\mathrm{IE}}=21 \mathrm{nS}, \tau_{\mathrm{E}}=55 \mathrm{~ms}, \rho=0.008, \tau_{\mathrm{p}}=$ $300 \mathrm{~ms}, \tau_{\mathrm{F}}=50 \mathrm{~ms}, \tau_{\mathrm{D}}=100 \mathrm{~ms}$.

in SM, the model response agrees with experiments qualitatively well even with PI alone, however, STP is needed to achieve quantitative agreement with experiments. On the other hand, even though a very strong STD may also cause the system to adapt (Fig. S2b), the fit of the STP-only model to experiments remains poor even by fine tuning the STF and STD strengths (see Fig. S2b-d in SM for details).

\section{Discussion}

In this study, we developed a simple neural circuit model for the antennal lobe of Drosophila and systematically studied the role of STP and PI in early odor information processing. Combining analytical derivations of a steady-state solution of the model and numerical simulations, we showed that the model can capture key characteristics of PNs' responses to different ORN inputs, in particular divisive normalization and adaptive responses to time-varying signals. Comparison with experimental results revealed that STP is crucial for the observed nonlinear divisive normalization, and the adaptive response to the timevarying ORN input is affected by both STP and PI with the latter playing a more dominant role in the experimental systems we 
bioRxiv preprint doi: https://doi.org/10.1101/2021.05.02.442289; this version posted May 2, 2021. The copyright holder for this preprint (which was not certified by peer review) is the author/funder, who has granted bioRxiv a license to display the preprint in perpetuity. It is made available under aCC-BY-NC-ND 4.0 International license.

analyzed.

Previous experiments have suggested that STD largely determines the nonlinear response function of PNs (9), and PI plays an important role in divisive normalization $(10,12)$. Our model extends these studies and showed that STP is crucial for the observed nonlinear divisive normalization. For stable ORN input, STF enhances PN's response while STD suppresses it. Yet, both STF and STD enhance the nonlinearity effect of the divisive normalization (Fig. 2). Although the transient response of PNs to step-like ORN input has been attributed to STD, our model shows that presynaptic inhibition predominately determines PN's response to more dynamic ORN input, such as triangle-shaped input patterns (Fig.5). In fact, our model can predict the response properties of PNs to arbitrary time-dependent ORN inputs. For example, when applying a set of sine-wave ORN inputs with different frequencies, our model predicts that the amplitude of PN's oscillatory response will increase as the frequency gets higher, while the time advance of PN's peak to ORN's peak will decrease (see Fig. S3 in SM). These predictions can be tested by future experiments.

Our model not only revealed the underlying mechanisms for the observed nonlinear divisive normalization behavior and adaptive responses to time-varying signals, it also provides a general framework for relating microscopic properties of the system such as time scales and strengths of STP and PI to macroscopic behaviors such as the input-output relation. As demonstrated in this work in the cases of VM7 and DL5 glomeruli, we can use our model to infer/estimate microscopic properties of the system from the measured input-output relation. More specifically, from our model study, the timescales of both STD $\left(\tau_{D}\right)$ and STF $\left(\tau_{F}\right)$ are predicted to be longer in DL5 than those in VM7, which can be verified by experiments (36). The model-based analysis of the input-output response can be extended to other glomeruli. Our model can also be used to make predictions for changes in the input-output relation when certain microscopic properties, e.g., the STP strength $\left(\tau_{F}\right.$ and $\left.\tau_{D}\right)$ or the PI strength $(\rho)$ are perturbed. These predictions can be tested in future experiments.

As we focused on building a minimal model to understand the underlying mechanism for nonlinear divisive normalization and adaptive response, we have made several simplifications in our study. At the synapse level, our model ignores the STP effect at the ORN-LN synapses (31), which can affect the LN response. In our circuit model, we only considered one type of LNs for simplicity. There are several type of LNs in the antennal lobe with diverse innervation patterns and physiological properties (29-31). For example, a small fraction of LNs are excitatory. Panglomerular LNs innervate all glomeruli with higher spontaneous firing rates than other LNs, they are inhibited or only weakly excited by odors. Such inhibition of panglomerular LNs tends to dis-inhibit the entire antannal lobe in the presence of odors (29). LNs also show distinct response dynamics to odors. Future study should address how these additional complexity and LN-LN interactions affect PN's response properties (37). Furthermore, although most PNs innervate a single glomerulus (uPNs) as we studied in this paper, some do receive input from multiple glomeruli (mPNs). uPNs and mPNs have different projection patterns and may carry different aspects of odor information to the higher brain regions such as lateral horn and mushroom body (38). Future studies that incorporate these important features (both at the synapse level and the network level) will further our understanding of the rich dynamics in the early olfactory information processing in Drosophila.

\section{ACKNOWLEDGEMENTS}

We thank Guangwei Si, Tianyi Wu and members of Tang lab for helpful discussion. The work by YT is supported by a NIH grant(R35GM131734).

\section{Bibliography}

1. Elissa A Hallem and John R Carlson. Coding of odors by a receptor repertoire. Cell, 125(1):143-160, 2006.

2. Harumi Saito, Qiuyi Chi, Hanyi Zhuang, Hiroaki Matsunami, and Joel D Mainland. Odor coding by a mammalian receptor repertoire. Science signaling, 2(60):ra9-ra9, 2009.

3. Guangwei Si, Jessleen K Kanwal, Yu Hu, Christopher J Tabone, Jacob Baron, Matthew Berck, Gaetan Vignoud, and Aravinthan DT Samuel. Structured odorant response patterns across a complete olfactory receptor neuron population. Neuron, 101(5):950-962, 2019.

4. Chih-Ying Su, Karen Menuz, and John R Carlson. Olfactory perception: receptors, cells, and circuits. Cell, 139(1):45-59, 2009.

5. Bill S Hansson and Marcus C Stensmyr. Evolution of insect olfaction. Neuron, 72(5):698-711, 2011.

6. Naoshige Uchida, Cindy Poo, and Rafi Haddad. Coding and transformations in the olfactory system. Annual review of neuroscience, 37:363-385, 2014.

7. Rachel I Wilson. Early olfactory processing in drosophila: mechanisms and principles. Annual review of neuroscience, 36:217-241, 2013.

8. Nicolas Y Masse, Glenn C Turner, and Gregory SXE Jefferis. Olfactory information processing in drosophila. Current Biology, 19(16):R700-R713, 2009.

9. Hokto Kazama and Rachel I Wilson. Homeostatic matching and nonlinear amplification at identified central synapses. Neuron, 58(3):401-413, 2008

10. Shawn R Olsen and Rachel I Wilson. Lateral presynaptic inhibition mediates gain control in an olfactory circuit. Nature, 452(7190):956-960, 2008.

11. Vikas Bhandawat, Shawn R Olsen, Nathan W Gouwens, Michelle L Schlief, and Rachel I Wilson. Sensory processing in the drosophila antennal lobe increases reliability and separability of ensemble odor representations. Nature neuroscience, 10(11):1474-1482, 2007.

12. Shawn R Olsen, Vikas Bhandawat, and Rachel I Wilson. Divisive normalization in olfactory population codes. Neuron, 66(2):287-299, 2010.

13. Matteo Carandini and David J Heeger. Normalization as a canonical neural computation. Nature Reviews Neuroscience, 13(1):51-62, 2012.

14. Sean X Luo, Richard Axel, and LF Abbott. Generating sparse and selective third-order responses in the olfactory system of the fly. Proceedings of the National Academy of Sciences, 107(23): 10713-10718, 2010

15. John Murlis, Joseph S Elkinton, and Ring T Carde. Odor plumes and how insects use them. Annual review of entomology, 37(1):505-532, 1992.

16. Neil J Vickers, Thomas A Christensen, Thomas C Baker, and John G Hildebrand. Odour-plume dynamics influence the brain's olfactory code. Nature, 410(6827):466-470, 2001.

17. Jonathan D Victor, Sebastian D Boie, Erin G Connor, John P Crimaldi, G Bard Ermentrout, and Katherine I Nagel. Olfactory navigation and the receptor nonlinearity. Journal of Neuroscience, 39 (19):3713-3727, 2019.

18. CT David, JS Kennedy, and AR Ludlow. Finding of a sex pheromone source by gypsy moths released in the field. Nature, 303(5920):804-806, 1983

19. Mahmut Demir, Nirag Kadakia, Hope D Anderson, Damon A Clark, and Thierry Emonet. Walking drosophila navigate complex plumes using stochastic decisions biased by the timing of odor encounters. Elife, 9:e57524, 2020.

20. Anmo J Kim, Aurel A Lazar, and Yevgeniy B Slutskiy. Projection neurons in drosophila antennal lobes signal the acceleration of odor concentrations. Elife, 4:e06651, 2015.

21. Katherine I Nagel and Rachel I Wilson. Biophysical mechanisms underlying olfactory receptor neuron dynamics. Nature neuroscience, $14(2): 208,2011$.

22. Anmo J Kim, Aurel A Lazar, and Yevgeniy B Slutskiy. System identification of drosophila olfactory sensory neurons. Journal of computational neuroscience, 30(1):143-161, 2011.

23. Carlotta Martelli and André Fiala. Slow presynaptic mechanisms that mediate adaptation in the olfactory pathway of drosophila. eLife, 8:e43735, 2019.

24. Charles F Stevens and Yanyan Wang. Facilitation and depression at single central synapses. Neuron, 14(4):795-802, 1995. 
bioRxiv preprint doi: https://doi.org/10.1101/2021.05.02.442289; this version posted May 2, 2021. The copyright holder for this preprint (which was not certified by peer review) is the author/funder, who has granted bioRxiv a license to display the preprint in perpetuity. It is made available under aCC-BY-NC-ND 4.0 International license.

25. Larry F Abbott, JA Varela, Kamal Sen, and SB Nelson. Synaptic depression and cortical gain control. Science, 275(5297):221-224, 1997.

26. Henry Markram, Yun Wang, and Misha Tsodyks. Differential signaling via the same axon of neocortical pyramidal neurons. Proceedings of the National Academy of Sciences, 95(9):5323-5328, 1998.

27. Jeremy S Dittman, Anatol C Kreitzer, and Wade G Regehr. Interplay between facilitation, depression, and residual calcium at three presynaptic terminals. Journal of Neuroscience, 20(4):1374-1385, 2000.

28. Yun Wang, Henry Markram, Philip H Goodman, Thomas K Berger, Junying Ma, and Patricia S Goldman-Rakic. Heterogeneity in the pyramidal network of the medial prefrontal cortex. Nature neuroscience, 9(4):534-542, 2006.

29. Ya-Hui Chou, Maria L Spletter, Emre Yaksi, Jonathan CS Leong, Rachel I Wilson, and Liqun Luo. Diversity and wiring variability of olfactory local interneurons in the drosophila antennal lobe. Nature neuroscience, 13(4):439, 2010

30. Katherine I Nagel, Elizabeth J Hong, and Rachel I Wilson. Synaptic and circuit mechanisms promoting broadband transmission of olfactory stimulus dynamics. Nature neuroscience, 18(1):56, 2015.

31. Katherine I Nagel and Rachel I Wilson. Mechanisms underlying population response dynamics in inhibitory interneurons of the drosophila antennal lobe. Journal of Neuroscience, 36(15):4325-4338, 2016.

32. Wulfram Gerstner, Werner M Kistler, Richard Naud, and Liam Paninski. Neuronal dynamics: From single neurons to networks and models of cognition. Cambridge University Press, 2014.

33. Danke Zhang, Yuanqing Li, and Si Wu. Concentration-invariant odor representation in the olfactory system by presynaptic inhibition. Computational and mathematical methods in medicine, 2013, 2013.

34. Danke Zhang, Si Wu, and Malte J Rasch. Circuit motifs for contrast-adaptive differentiation in early sensory systems: the role of presynaptic inhibition and short-term plasticity. PloS one, 10(2), 2015.

35. Misha Tsodyks, Klaus Pawelzik, and Henry Markram. Neural networks with dynamic synapses. Neural computation, 10(4):821-835, 1998.

36. LF Abbott, K Sen, JA Varela, J Gibson, and SB Nelson. Functional consequences of synaptic depression for response properties in v1. In Soc. Neurosci. Abstr, volume 22, page 952, 1996.

37. Kuo-Wei Kao and Chung-Chuan Lo. Short term depression, presynaptic inhibition and local neuron diversity play key functional roles in the insect antennal lobe. Journal of computational neuroscience, 48:213-227, 2020

38. Alexander Shakeel Bates, Philipp Schlegel, Ruairi JV Roberts, Nikolas Drummond, Imaan FM Tamimi, Robert Gillies Turnbull, Xincheng Zhao, Elizabeth C Marin, Patricia Demetria Popovici, Serene Dhawan, et al. Complete connectomic reconstruction of olfactory projection neurons in the fly brain. BioRxiv, 2020. 


\section{Supplementary Material}

A. The linear relation between firing rate and conductance. In the main text, we have used the approximation that a neuron's firing rate is proportional to its conductance $\left(R_{P N / L N}=k g_{P N / L N}\right)$ at steady state. Here, we numerically verify the validity of this approximation. Consider a leaky integrate-and-fire neuron whose membrane potential evolves as

$$
\tau_{m} \frac{d V}{d t}=V_{L}-V+r_{m} g_{E}\left(V-V_{E}\right)
$$

for $V<V_{t h}$. Here $V_{L}$ and $V_{E}$ are the resting potential and the reverse potential respectively, $r_{m}$ is the membrane resistance, $g_{E}$ is the conductance, $\tau_{m}$ is the membrane time constant and $V_{t h}$ is the firing threshold. When $V$ reaches $V_{t h}$, the membrane potential is reset to $V_{L}$. Initializing $V$ at the resting potential, i.e., $V(t=0)=V_{L}$, the solution of Eq. (11) is given by

$$
V(t)=\frac{V_{L}}{r_{m} g_{E}-1}\left[r_{m} g_{E} e^{-\left(1-r_{m} g_{E}\right) t / \tau_{m}}-1\right]
$$

We are interested in the time required $\Delta t$ for $V(t)$ to reach the firing threshold $V_{t h}$ from the resting potential $V_{L}$. Assuming that $t \leq \Delta t \ll \tau_{m}$, we can expand Eq. (12) linearly in $t / \tau_{m}$ :

$$
V(t)=V_{L}+\frac{E_{L} r_{m} g_{E} t}{\tau_{m}}
$$

$\Delta t$ can be solved from above equation:

$$
\Delta t=\frac{\left(V_{t h}-V_{L}\right) \tau_{m}}{V_{L} r_{m} g_{E}}
$$

The firing rate of the neuron is defined by how many times $V$ reaches $V_{t h}$ in a unit time

$$
R=\frac{1}{\Delta t}=\frac{V_{L} r_{m} g_{E}}{\left(V_{\mathrm{th}}-V_{L}\right) \tau_{m}}=k g_{E}
$$

which is proportional to the conductance $g_{E}$. The above approximation is further verified by numerical simulation (Fig. S1).

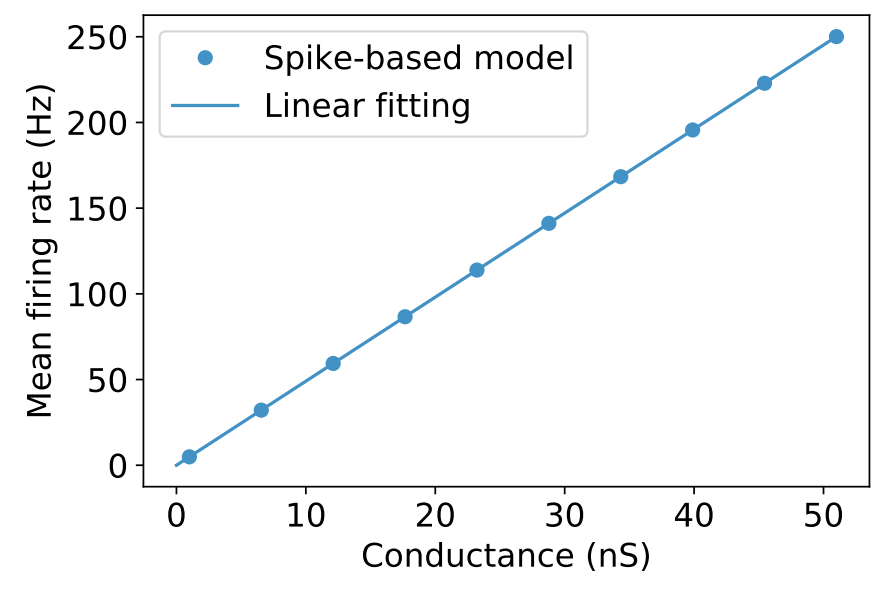

Fig. S1. Firing rate is proportional to the steady state conductance $\left(g_{E}\right)$ in the leaky integrate-and-fire model. The parameters in Eq.11 used for the simulation are: $\tau_{m}=20 \mathrm{~ms}, V_{L}=-70 \mathrm{mV}, V_{E}=0 \mathrm{mV}, V_{t h}=-50 \mathrm{mV}, r_{m}=3.3 \times 10^{7} \Omega$.

B. Response of PNs to triangular-shaped firing rates of ORNs. In this section, we describe in detail the approximation used in the calculations when we study PN's response to triangular-shaped inputs. First, we consider a simpler scenario, where ORN's firing rate increases linearly without bound, i.e., $R=K t$. Then, Eq.(1-5) in the main text become 


$$
\begin{aligned}
\frac{d R_{L N}}{d t} & =-\frac{R_{L N}}{\tau_{E}}+k \omega^{I E} K t \\
\tau_{p} \frac{d p}{d t} & =-p+\frac{1}{1+\rho R_{L N}}, \\
\frac{d R_{P N}(t)}{d t} & =-\frac{k g_{P N}}{\tau_{E}}+k \omega^{E E} u^{+} x p K t \\
\frac{d x}{d t} & =\frac{1-x}{\tau_{D}}-x u^{+} p K t \\
\frac{d u^{-}}{d t} & =-\frac{u^{-}}{\tau_{F}}+U\left(1-u^{-}\right) p K t, \\
u^{+} & =u^{-}+U\left(1-u^{-}\right) .
\end{aligned}
$$

To analyze the adaptive behavior of PN responses, we try to give an approximate solution of the above equations. These equations can be separated into two groups based on the biological mechanisms they describe: the first two equations describe presynaptic inhibition and the rest equations are related to STP.

The presynaptic inhibition terms. Solving the first two equations of Eq. (16) we obtain:

$$
\begin{aligned}
R_{L N}(t) & =k \omega^{I E} K \tau_{E}\left[t-\tau_{E}\left(1-e^{-\frac{t}{\tau_{E}}}\right)\right], \\
p(t) & =e^{-\frac{t}{\tau_{p}}}+e^{-\frac{t}{\tau_{p}}} \int_{0}^{t} \frac{e^{\frac{t^{\prime}}{\tau_{p}}} d t^{\prime}}{\tau_{p}\left\{1+A K\left[t^{\prime}-\tau_{E}\left(1-e^{-\frac{t^{\prime}}{\tau_{E}}}\right)\right]\right\}}
\end{aligned}
$$

with initial conditions $p(0)=1$ and $R_{L N}(0)=0$. Here $A=k \rho \tau_{E} \omega^{I E}$, as defined previously in the steady state solution in the main text. Since the plateau appears at $t \gg \tau_{E}$, the integrand of Eq. (18) can be approximated as

$$
\frac{e^{\frac{t^{\prime}}{\tau_{p}}}}{\tau_{p}\left\{1+A K\left[t^{\prime}-\tau_{E}\left(1-e^{-\frac{t^{\prime}}{\tau_{E}}}\right)\right]\right\}} \approx \frac{e^{\frac{t^{\prime}}{\tau_{p}}}}{\tau_{p}\left(1-A K \tau_{E}\right)+\tau_{p} A K t^{\prime}}
$$

Plug it back into Eq. (18), we obtain:

$$
p(t) \approx e^{-\frac{t}{\tau_{p}}}+\frac{e^{-\left(\frac{t}{\tau_{p}}+\frac{1-A K \tau_{E}}{A K \tau_{p}}\right)}}{A K \tau_{p}}\left[\operatorname{Ei}\left(\frac{t}{\tau_{p}}+\frac{1-A K \tau_{E}}{A K \tau_{p}}\right)-\operatorname{Ei}\left(\frac{1-A K \tau_{E}}{A K \tau_{p}}\right)\right],
$$

where $\operatorname{Ei}(x)=\int_{-\infty}^{x} \frac{e^{x^{\prime}}}{x^{\prime}} d x^{\prime}$ denotes the exponential integral. Asymptotically expand $\operatorname{Ei}(x)$ as $\operatorname{Ei}(x)=\frac{e^{x}}{x} \sum_{n=0}^{N-1} \frac{n !}{x^{n}}$ and consider the limit $t \rightarrow \infty$, we have

$$
p(t) \approx \frac{1}{A K\left(t-\tau_{E}\right)+1}-\frac{A K \tau_{E}}{1-A K \tau_{E}} e^{-\frac{t}{\tau_{p}}}
$$

Multiply Eq. (21) by $K t$ gives the effective input $R_{\text {eff }}$ Eq. (9) in main text.

The STP terms. In this section we consider the last 4 equations in Eq. (16) which describe the STP mechanism. As we discussed in the main text, for $t \gg \tau_{p}, p \approx(A R(t))^{-1}$, which leaves the effective input $R_{\text {eff }}=p(t) R(t)$ approximately constant in time. We use this approximation to investigate the plateau behavior of PN response. Plugging it back into Eq. (16), we have $u^{-}(t)=\tau_{u} U R_{\mathrm{eff}}\left(1-e^{-\frac{t}{\tau_{u}}}\right)$ where $1 / \tau_{u} \equiv 1 / \tau_{F}+R_{\mathrm{eff}} U$.

The equation for $x$ becomes:

$$
\frac{d x}{d t}+\left[\frac{1}{\tau_{x}}-(1-U) U R_{\mathrm{eff}}^{2} \tau_{u} e^{-\frac{t}{\tau_{u}}}\right] x=\frac{1}{\tau_{D}},
$$

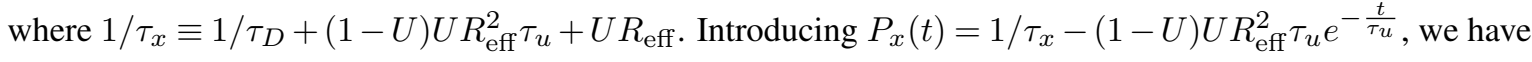

$$
x(t)=e^{-\int_{0}^{t} P_{x}\left(t^{\prime}\right) d t^{\prime}}+e^{-\int_{0}^{t} P_{x}\left(t^{\prime}\right) d t^{\prime}} \int_{0}^{t} \frac{1}{\tau_{D}} e^{\int_{0}^{t^{\prime}} P_{x}\left(t^{\prime \prime}\right) d t^{\prime \prime}} d t^{\prime} .
$$


bioRxiv preprint doi: https://doi.org/10.1101/2021.05.02.442289; this version posted May 2, 2021. The copyright holder for this preprint (which was not certified by peer review) is the author/funder, who has granted bioRxiv a license to display the preprint in perpetuity. It is made available under aCC-BY-NC-ND 4.0 International license.

C Model variants incorporating different mechanisms revealing the role of STP in response to time-varying signals

Since we are interested in the plateau behavior of PNs, where $t \gg \tau_{u}, \tau_{x}$. We can approximate $\int_{0}^{t} P_{x}\left(t^{\prime}\right) d t^{\prime}$ as $\frac{t}{\tau_{x}}$. Plug it back to Eq. (23) and neglect higher order corrections, we have $x(t) \approx \tau_{x} / \tau_{D}$. Similarly,

$$
u^{+}(t) x(t)=(1-U) u^{-}(t) x(t)+U x(t) \approx \frac{\tau_{x}}{\tau_{D}}\left[(1-U) U \tau_{u} R_{\mathrm{eff}}+U\right] .
$$

The magnitude of the plateau response of PN can be approximated by its steady state activity for large $t$,

$$
R_{P N}(t)=k \omega^{E E} \tau_{E} R_{\mathrm{eff}} u^{+}(t) x(t) \approx k \omega^{E E} \tau_{E} R_{\mathrm{eff}} \frac{\tau_{x}}{\tau_{D}}\left[(1-U) U \tau_{u} R_{\mathrm{eff}}+U\right]
$$

where $R_{\text {eff }}=p(t) K t \approx 1 / A$ with $p(t)$ given by Eq. (21). We see that the plateau magnitude of $R_{P N}$ is related to $R(t)$ only through $R_{\text {eff }}$, which is the final value that $p R(t)$ will reach. It is independent of $t$ and $K$, therefore the magnitude of PN response plateau is not affected by the input changing rate $K$, but only depends on properties that are intrinsic to the network.

C. Model variants incorporating different mechanisms revealing the role of STP in response to time-varying signals. In this section, we compare several model variant incorporating only part of the mechanisms compared to the full model (standard model) in the main text. As shown in Fig. S2, PN response with only presynaptic inhibition is slightly different from the standard model with both STP and presynaptic inhibition, while the STP only model deviates from the standard model more significantly. In addition, as shown in Fig. S2(b), the STP-only model can not distinguish the experimentally observed different plateau heights for different inputs. We conclude that presynaptic inhibition plays a dominant role in shaping a PN's response to time varying signals while STP fine tunes the adaptive response.
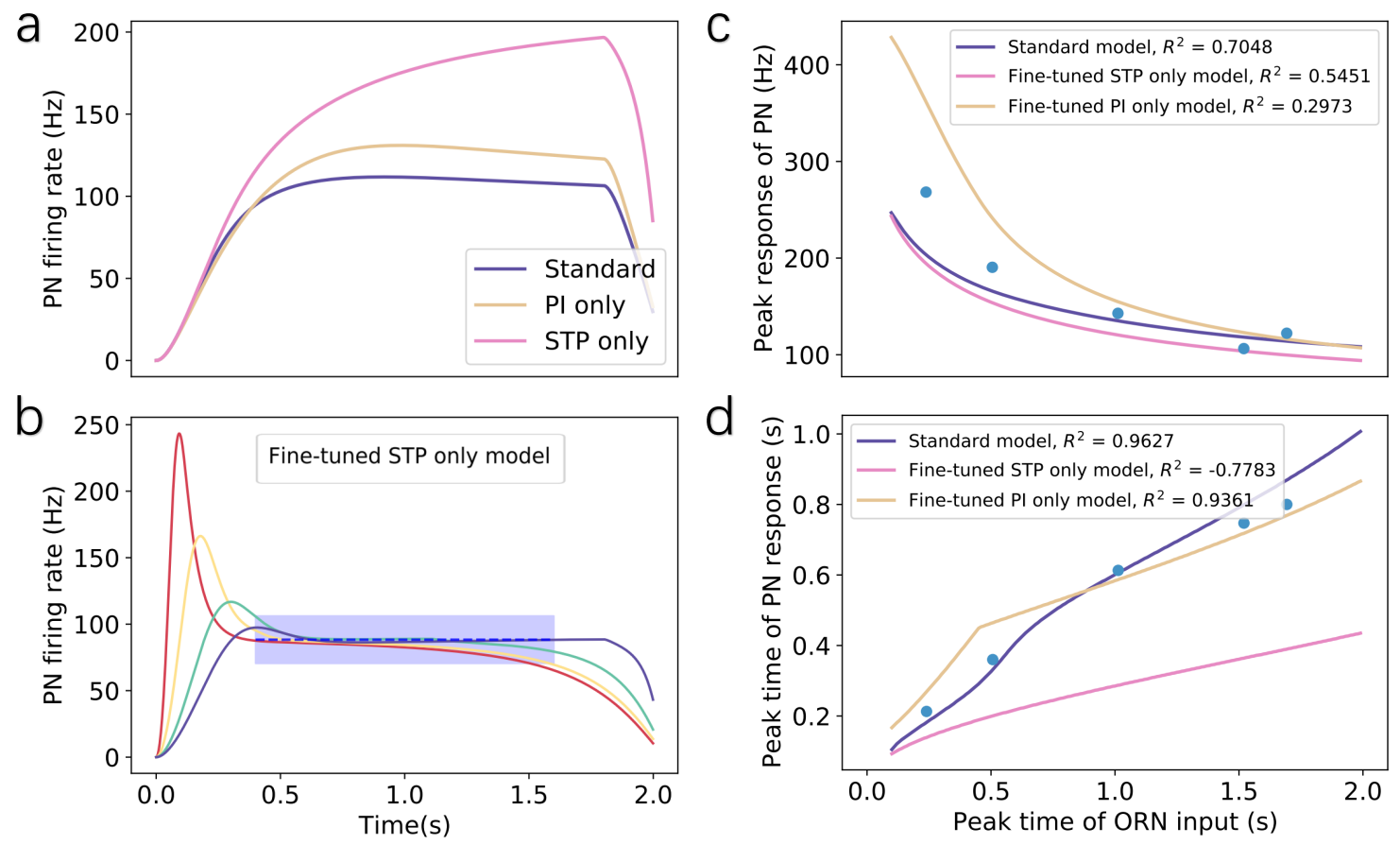

Fig. S2. PN response under different conditions reveals the interaction between STP and presynaptic inhibition (PI). (a) PN response in three model variants to triangle-shaped ORN input as in Fig.5 with peak time at $1.8 \mathrm{~s}$ and peak amplitude $120 \mathrm{~Hz}$. All the three models share the same set of parameters as in Fig. 5, except that the PI only model has $\tau_{D}=\tau_{F}=0$ and the STP only model has $\rho=0$. (b)The best fit to the experiments by using the STP only model. For all model variants, the parameters are selected to best fit the plateau height (shaded region) for the slowest input. Comparing the fitting of three model variants (standard, STP only, and PI only) with experimentally observed peak response (c) and peak response time (d), we found the standard model performs much better than other model variants. Note that the $R^{2}$ value for the STP only model is negative, which means that the STP-only model fails to fit the peak time data when it is tuned to fit the plateau height as shown in (b). Parameters in the variant models that are different from the standard model are: $\tau_{D}=270 \mathrm{~ms}, \tau_{F}=100 \mathrm{~ms}, \rho=0, U=0.2, \omega^{E E}=90 \mathrm{nS}$ for the STP-only model; $\tau_{D}=0 \mathrm{~ms}, \tau_{F}=0 \mathrm{~ms}$, $\rho=0.01, \tau_{p}=250 \mathrm{~ms}, U=0.25, \omega^{E E}=80 \mathrm{nS}$ for the PI-only model. 
bioRxiv preprint doi: https://doi.org/10.1101/2021.05.02.442289; this version posted May 2, 2021. The copyright holder for this preprint (which was not certified by peer review) is the author/funder, who has granted bioRxiv a license to display the preprint in perpetuity. It is made available under aCC-BY-NC-ND 4.0 International license.
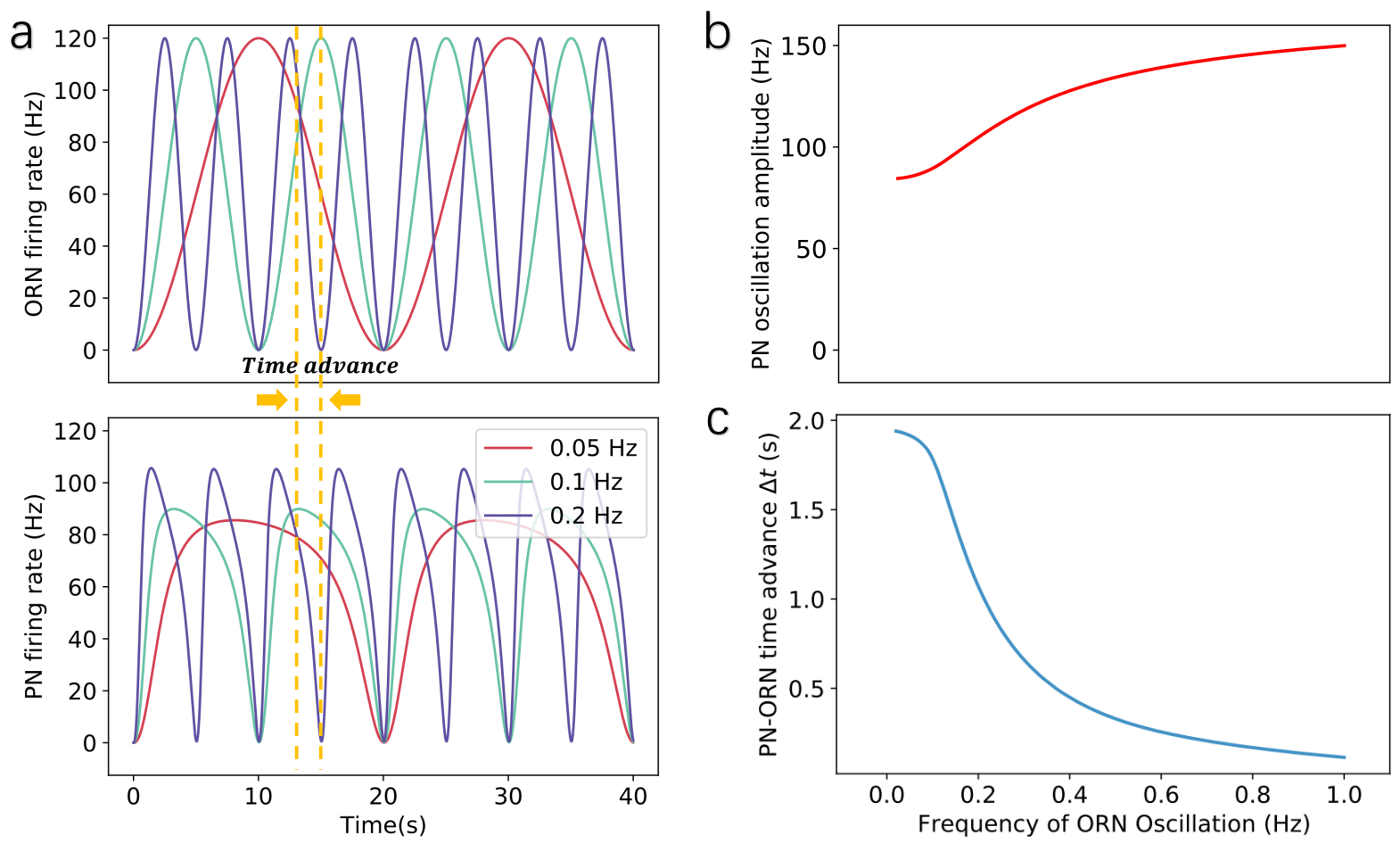

Fig. S3. Prediction of PN response to oscillatory ORN inputs. (a) Upper panel: Simulated sine-wave ORNs firing rates with different frequency. The peak firing rates are the same for all inputs. Lower panel: responses of PNs to sine-wave ORNs inputs. The two dotted lines mark the peak of ORN input and its corresponding PN output with a frequency of $0.1 \mathrm{~Hz}$. The gap between these dotted lines is defined as the time advance $\Delta t$ of PN's response. (b) The peak responses of PNs increase with the frequency of ORN oscillation. (c) PN's response always reaches to a peak earlier than that of the input signal, but the time advance $\Delta t$ decreases as the oscillation frequency increase. Parameters used in the model are the same as in Fig. 5 